\title{
Diseño de certificación lingüística comunicativa: ¿cómo evaluar la expresión e interacción escritas y orales?
}

\author{
Marta Baralo \\ Universidad Nebrija \\ mbaralo@nebrija.es
}

Resumen: La posibilidad de medir y de evaluar las actividades de expresión y de interacción en una lengua, sea por el canal oral o el escrito, es un desafío para los especialistas en lingüística aplicada pues se trata de una apreciación subjetiva, que depende en gran parte del evaluador, de sus creencias y actitudes. Para conseguir que la prueba de evaluación sea válida, fiable y auténtica es necesario que su diseño esté sujeto a un conjunto de parámetros de calidad establecidos por las agencias e instituciones especializadas y que su aplicación cuente con un conjunto de herramientas de evaluación suficientemente probadas y un conjunto de evaluadores preparados que aseguren el acuerdo en la valoración del desempeño lingüístico comunicativo de los candidatos. Se presenta aquí la experiencia investigadora en el marco del diseño e implantación del Diploma LETRA (Lengua Española para Trabajadores inmigrantes) en la Comunidad de Madrid ${ }^{1}$.

Palabras clave: certificación lingüística, evaluación, competencia comunicativa, expresión e interacción escritas y orales, instrumentos de evaluación subjetiva, descriptores, español para inmigrantes.

1. El Proyecto de implantación de un programa de formación y certificación lingüística para trabajadores inmigrantes en la Comunidad de Madrid ha sido financiado, de 2008 a 2011, por la Consejería de Inmigración y Cooperación de la Comunidad de Madrid y por la Fundación Antonio de Nebrija. 


\begin{abstract}
It is a real challenge for specialists in applied linguistics to measure and evaluate activities of language expression and interaction, no matter whether an oral or written channel is used for this purpose. In any case, the evaluation mainly depends on the evaluator's subjective appreciation, his/ her beliefs and attitudes. In order to achieve a valid, reliable and authentic evaluation test, its design is required to be subject to a set of quality parameters, established by specialized institutions and agencies. Furthermore, its application needs to count on a group of trained evaluators that may guarantee agreement in the valuation of the candidate's communicative and linguistic performance. This paper presents the research experience carried out within the framework of the design and implementation of the Diploma LETRA (Lengua Española para Trabajadores inmigrantes) in the Community of Madrid.
\end{abstract}

Keywords: language certification, evaluation, communicative competence, oral and written language expression and interaction, tools for subjective evaluation, descriptors, Spanish for immigrants. 


\section{Objetivos y marco de aplicación}

En este artículo presentamos una síntesis del marco de aplicación, de los fundamentos teóricos, de los criterios de evaluación y de las decisiones tomadas por el equipo investigador para el diseño de las pruebas de expresión e interacción escritas y orales de un examen de certificación lingüística comunicativa de español para trabajadores inmigrantes ${ }^{2}$. Este proyecto se ha desarrollado con el propósito de proporcionar un currículo específico para una certificación que responda a las necesidades comunicativas reales de trabajadores inmigrantes, no atendidos por las instituciones académicas, que recurren a diferentes ONGs, a instituciones sociales.

Después de realizar una serie de estudios de necesidades lingüísticas comunicativas entre diferentes actores implicados en la enseñanza y en la comunicación en ámbitos laborales y administrativos de los trabajadores inmigrantes hablantes no nativos de español, se ha fijado un nivel A2-n de dominio de la lengua española y se ha asumido una marcada asimetría en el dominio de cada una de las subcompetencias lingüísticas. Lo más importante y llamativo ha sido conseguir el alcance léxico requerido en los entornos laborales específicos más frecuentes de la inmigración en Madrid, un alto grado de competencia pragmática funcional con especial atención a la cortesía, así como un bajo nivel de exigencia de corrección gramatical, reducida a lo estrictamente necesario para posibilitar la comunicación.

Específicamente, en este trabajo vamos a presentar las dificultades que ofrece una evaluación subjetiva para conseguir asegurar un alto grado de validez, de fiabilidad y de viabilidad en el diseño de unas pruebas de expresión y de interacción que dependen en parte del propio evaluador del candidato y de variadas condiciones y factores individuales y contextuales. Así mismo, queremos compartir las decisiones tomadas para la evaluación de la expresión e interacción escritas y orales en el diseño de una certificación lingüística, con la presentación de los instrumentos diseñados y de los resultados obtenidos en la primera convocatoria realizada para 300 candidatos.

Asumimos que el español para trabajadores inmigrantes hablantes de otras lenguas no se puede considerar una lengua de especialidad, sino que se trata de la lengua general, pero en contextos específicos de uso, los laborales y administrativos. Al mismo tiempo necesitamos definir cuáles son los contenidos que se deben incluir en un currículo específico para una certificación de nivel inicial,

2. Todo el trabajo ha sido realizado por el Grupo de investigación LAELE (Lingǘstica aplicada a la enseñanza de lenguas) de la Universidad Nebrija, integrante del Grupo INMIGRA i+d de la Comunidad de Madrid. Se puede consultar en $<$ http://www.grupoinmigra-imasd.es $>$. 
partiendo de los descriptores de los niveles de referencia A1 y A2 del Marco común europeo de referencia para la enseñanza, el aprendizaje y la evaluación de las lenguas - MCER - y de los contenidos referidos en el Pan Curricular del IC para ambos niveles. Sin embargo, el estudio comparativo de estos contenidos con las necesidades reales de comunicación detectadas en la fase etnográfica del análisis previo al diseño pusieron en evidencia la necesidad de un diseño curricular específico.

Como hemos indicado en un estudio previo (Escudero y Guerra 2009; Baralo y Estaire 2011), la oferta didáctica para adultos que desean aprender español es muy variada en el ámbito institucional, en las diferentes administraciones públicas, en las instituciones benéficas y entre el voluntariado. En los últimos años numerosos investigadores de diferentes procedencias — didácticas, lingüísticas e interculturales - se han ocupado de promover investigaciones que han permitido analizar y describir las necesidades sociolingüísticas específicas de este colectivo, diseñar programas y cursos de español para trabajadores inmigrantes, editar material didáctico en soportes convencionales y electrónicos. Buena muestra de toda esta actividad docente e investigadora se encuentra recogida en documentos como las Conclusiones del III Encuentro de especialistas de enseñanza de segundas lenguas a inmigrantes (Universidad de Granada 2008) ${ }^{3}$.

Aplicando los principios del MCER, hemos diseñado unas tareas de evaluación de la expresión y de la interacción orales y escritas que garanticen una cierta autonomía comunicativa en contextos laborales a los inmigrantes adultos que deseen aprender español. Los fundamentos y las razones de esta propuesta son variados y de diferente carácter, pero podemos resumirlos en la oportunidad, la motivación y utilidad que proporciona la obtención de una certificación. Buena muestra de esta necesidad de certificación son los enunciados que se recogen en documentos concensuados por especialistas como los siguientes:

Manifiesto de Santander: 2. Reivindicamos un plan general de organización, normalización, regulación y evaluación de la enseñanza de segundas lenguas a personas inmigrantes y refugiados. (MS)

Propuestas de Alicante: 1. Es necesaria y prioritaria la redacción de currículos de enseñanza de L2 adaptados al Marco Común Europeo de Referencia para las Lenguas (MCER). 2. Hay que diseñar acreditaciones (certificados) específicas para los cursos de enseñanza de L2 que unifiquen las existentes y que puedan ser expedidas por las diferentes instituciones y organizaciones que imparten los cursos de idiomas. 3. Estas acreditaciones tendrán un valor informativo y en ellas deberá recogerse: la cantidad de horas cursadas y el nivel alcanzado, así

3. Dos antecedentes esenciales a este documento son el Manifiesto de Santander-MS- (2004) y Propuestas de Alicante - PA- (2006). 
como una pequeña descripción de los contenidos que se han trabajado en el curso, según los descriptores del MCER.

Este artículo se centra solo en dos aspectos de la competencia comunicativa de un hablante plurilingüe: desenvolverse con eficacia en situaciones de expresión y de interacción comunicativa intercultural, oral y escrita, en el ámbito laboral y adminsitrativo. En estas situaciones, el hablante no nativo necesita comprender y hacerse entender en actos lingüísticos en los que debe codificar en la lengua nueva sus conocimientos y emociones, que forman parte de su experiencia de vida o de enseñanzas recibidas en su lengua materna. Es decir, debe activar su competencia léxica, y lingüística en general, para poder comunicarse. Creemos que un buen conocimiento sociocultural de la comunidad lingüística meta, aunque se posea un nivel de dominio bajo de su lengua, puede ayudar a resolver la situación comunicativa con eficacia. Tal como indica el MCER y asumimos en este trabajo, este desequilibrio entre la competencia lingüística y la pluricultural se puede dar, inclusive en la propia lengua y cultura nativas, ya que un hablante puede estar expuesto a distintos dialectos con sus propias normas y diferencias léxicas y socioculturales. Las competencias plurilingüe y pluricultural tienen un carácter variable e inestable, por su propia definición de interlengua y de intercultura, caracterizadas por una configuración transitoria y cambiante, a diferencia del carácter firme y estable de las competencias nativas (MCER 6.1.2).

La realidad de los inmigrantes no es uniforme; varía en función del tiempo que llevan en el lugar, de la lengua materna, de las relaciones sociales en las que se insertan. Es la competencia comunicativa la que le permite relacionarse con los servicios públicos, con las ofertas laborales, con los sistemas de participación, etc., que redunda en su propio beneficio y en la igualdad de oportunidades con respecto a los demás miembros ya integrados en el funcionamiento de las dinámicas públicas de nuestra sociedad.

\section{La expresión e interacción comunicativas}

Según el Marco común europeo de referencia, el usuario básico de nivel A2:

Es capaz de comprender frases y expresiones de uso frecuente relacionadas con áreas de experiencia que le son especialmente relevantes (información básica sobre sí mismo y su familia, compras, lugares de interés, ocupaciones, etc.). Sabe comunicarse a la hora de llevar a cabo tareas simples y cotidianas que no requieran más que intercambios sencillos y directos de información sobre cuestiones que le son conocidas o habituales. Sabe describir en términos sencillos aspectos de su pasado y su entorno así como cuestiones relacionadas con sus necesidades inmediatas. (MCER 6.1.2.) 
El perfil lingüístico específico que proponemos supone que el desarrollo de las destrezas sea irregular; aunque todas las capacidades que integran la competencia lingüística comunicativa tienen que ser utilizadas por el usuario de la lengua para abordar con eficacia toda la serie de actos comunicativos, no todos los trabajadores inmigrantes querrán o necesitarán adquirirlas en la lengua española. Por ejemplo, algunos inmigrantes no necesitarán la lengua escrita, mientras que a otros puede que les interese precisamente la comprensión y producción de textos escritos. Sin embargo, eso no implica necesariamente que dichos hablantes vayan a limitarse a las formas hablada y escrita de la lengua, respectivamente.

Debido a este desequilibrio, una de las características de la competencia plurilingüe y pluricultural es que, a la hora de aplicar esta competencia, el individuo en cuestión utiliza sus destrezas y conocimientos tanto generales como lingüísticos (véanse los capítulos 4 y 5 del MCER) de diferentes formas. Por ejemplo, las estrategias utilizadas para llevar a cabo tareas que suponen el uso de la lengua pueden variar según la lengua en cuestión. La competencia existencial (saber ser), que demuestra la apertura, la sociabilidad y la buena voluntad mediante el uso de gestos, mímica y proxémica, puede, en el caso de una lengua en la que el individuo tiene un nivel pobre del componente lingüístico, compensar esta insuficiencia durante el curso de la interacción con un hablante nativo, mientras que en una lengua que conoce mejor, este mismo individuo puede adoptar una actitud más lejana o reservada. La tarea puede que también se vuelva a definir y el mensaje lingüístico se reforme o se redistribuya, según los recursos disponibles para la expresión o la percepción que el individuo tiene de estos recursos. Se trata de una competencia diferenciada que permite el cambio de lengua cuando la situación de comunicación lo permite, ya que la competencia plurilingüe y pluricultural no se compone de la simple adición de competencias monolingües, sino que permite combinaciones y alternancias de distinto tipo. Es posible cambiar de código durante el mensaje o recurrir a formas bilingües de habla. Esta competencia también fomenta el desarrollo de la consciencia sobre la lengua y la comunicación, e incluso de las estrategias metacognitivas que le permiten avanzar en su capacidad comunicativa y aprovechar competencias sociolingüísticas y pragmáticas preexistentes que a su vez desarrolla más. Esto ocurre aunque la competencia plurilingüe sea «desequilibrada» y el dominio de la lengua española sea «parcial».

\section{Prueba de expresión e interacción escritas}

La evaluación del nivel de dominio de estas competencias comunicativas se ha realizado mediante tres tareas específicas, con una duración de 20 minutos en total, que tienen sus propias especificaciones. La Tarea 1 ha sufrido un proceso de 
diseño largo y con varias modificaciones a lo largo del proceso de pilotaje. Como se puede ver en la Tabla 1 se trata de rellenar un impreso con datos personales, lo que resultaba ser un área de extrema sensibilidad para quienes se sentían expuestos a un control de tipo policial con las preguntas sobre sí mismos. Para evitar esta variable emocional se introdujo la figura de un tercero, sobre el que se han de seleccionar datos dados.

Esta es la única prueba de expresión que se puede medir de manera objetiva, cuantitativa, según los huecos del formulario que rellene satisfactoriamente, ya que se trata de una expresión controlada y delimitada por el propio carácter de este género discursivo. Desde el punto de vista de la escritura se valora de manera ponderada que la letra sea legible; de lo contrario, se considera no válido el ítem que no se entienda.

El diseño de la Prueba se encuentra en la Figura 1.

\section{Tabla 1. Especificaciones de la TAREA 1 de la Prueba de expresión e interacción escritas: formulario}

\begin{tabular}{|c|c|c|c|}
\hline Tarea & Estímulo inicial & $\begin{array}{c}\text { Formato } \\
\text { de la prueba }\end{array}$ & $\begin{array}{c}\text { Capacidades } \\
\text { evaluadas }\end{array}$ \\
\hline $\begin{array}{l}\text { Tarea } 1 \\
\text { Rellenar un formu- } \\
\text { lario o documento } \\
\text { con datos perso- } \\
\text { nales incluidos en } \\
\text { un cuadro (ámbito } \\
\text { administrativo o } \\
\text { laboral). }\end{array}$ & $\begin{array}{l}\text { + Formulario o do- } \\
\text { cumento en el que se } \\
\text { solicitan datos perso- } \\
\text { nales y/o laborales. } \\
\text { + Datos personales } \\
\text { y/o laborales de una } \\
\text { persona, con los que } \\
\text { se deberá rellenar el } \\
\text { formulario o docu- } \\
\text { mento. }\end{array}$ & $\begin{array}{l}\text { El examinando } \\
\text { deberá rellenar un } \\
\text { formulario en el que } \\
\text { se solicita infor- } \\
\text { mación personal } \\
\text { básica, utilizando } \\
\text { datos incluidos en } \\
\text { un cuadro. }\end{array}$ & $\begin{array}{l}\text { El examinando } \\
\text { PUEDE: } \\
\text { Rellenar por escrito } \\
\text { un formulario } \\
\text { sencillo del ámbito } \\
\text { administrativo, con } \\
\text { los datos personales } \\
\text { incluidos en un } \\
\text { cuadro (nombre, } \\
\text { apellidos, lugar y } \\
\text { fecha de nacimiento, } \\
\text { dirección, NIE, } \\
\text { etc.). }\end{array}$ \\
\hline
\end{tabular}


Figura 1. Tarea 1 de expresión e interacción escritał rellenar un formulario

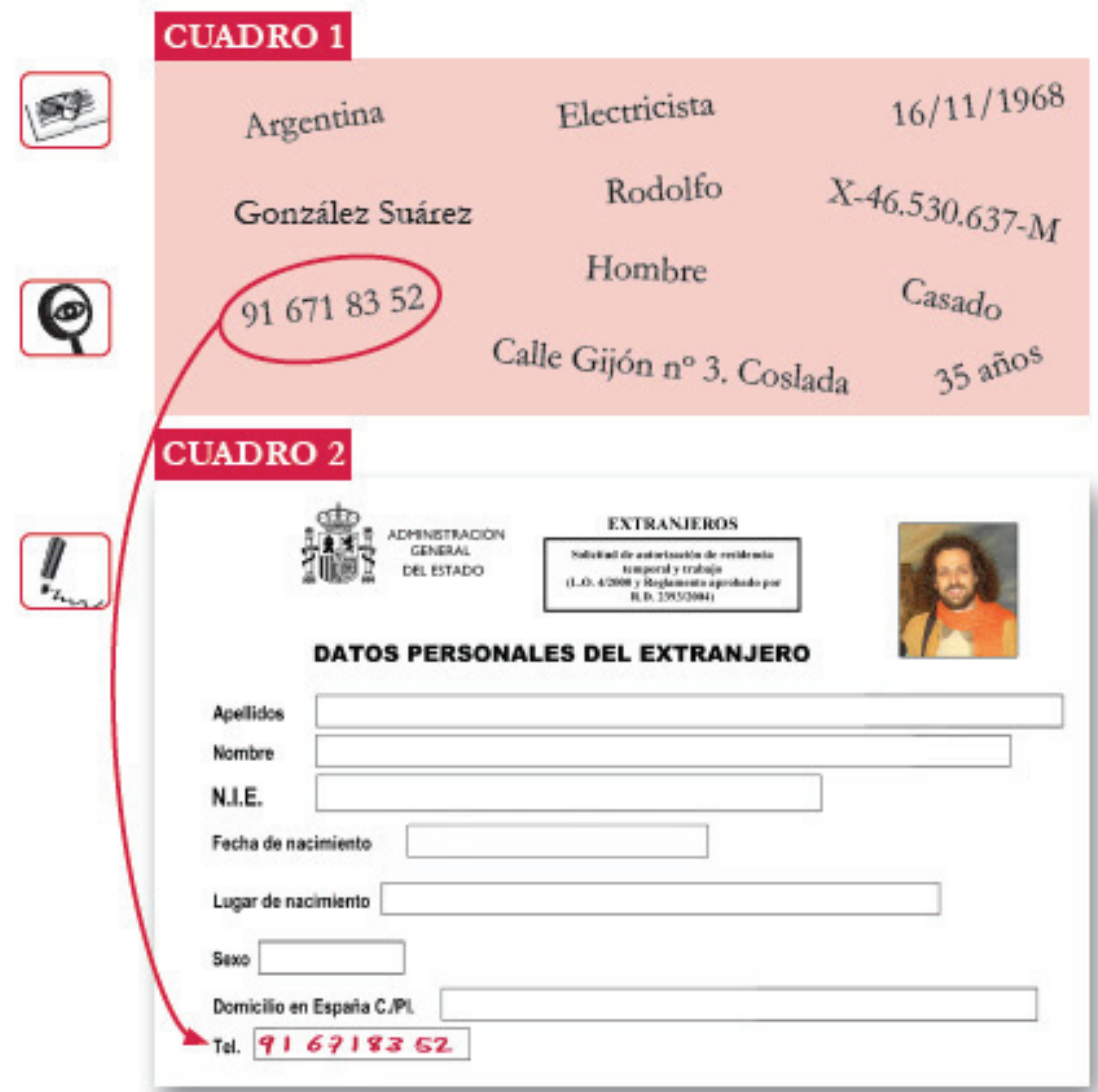

Las Tareas 2 y 3 de la Prueba de expresión e interacción escrita, al igual que las tareas orales constituyen pruebas de evaluación subjetivas, que evalúan el desempeño lingüístico comunicativo del candidato. Se trata de diagnosticar el nivel de dominio de la lengua, de forma cualitativa y cuantitativa, para comprobar en una situación de comunicación simulada en el acto del examen la eficacia que consigue en el acto de comunicación, mediante el uso escrito y oral de la lengua de manera funcional. Para poder hacer esta medición de manera válida y fiable se diseñaron y pilotaron con casi 200 inmigrantes, dos tareas que consisten en redactar dos textos adecuados a la situación de comunicación planteada. Para asegurar la garantía de fiabilidad se establece la necesidad de realizar dos evaluaciones de cada prueba por parte de dos evaluadores diferentes que utilicen una herramienta diferente para una evaluación diferente y complementaria, una holística y otra analítica. 
La Tarea 2 consiste en la redacción de un anuncio, atendiendo a todas las características del género indicadas en la situación, como se puede ver en las especificaciones de la Tabla 2 y en la muestra de la tarea en la Figura 2, a continuación:

\section{Tabla 2. Especificaciones Tarea 2 de la Prueba de expresión e interacción escritas anuncio}

\begin{tabular}{|c|c|c|c|}
\hline Tarea & Estímulo inicial & $\begin{array}{c}\text { Formato } \\
\text { de la prueba }\end{array}$ & $\begin{array}{c}\text { Capacidades } \\
\text { evaluadas }\end{array}$ \\
\hline $\begin{array}{l}\text { Tarea } 2 \\
\text { Escribir un } \\
\text { anuncio ofertan- } \\
\text { do o solicitando } \\
\text { algo (ámbito } \\
\text { laboral, público o } \\
\text { personal) }\end{array}$ & $\begin{array}{l}\text { + Finalidad / } \\
\text { contexto } \\
\text { + Lista de puntos } \\
\text { que se deben in- } \\
\text { cluir en el anuncio } \\
\text { + Espacio en blan- } \\
\text { co en un tablón } \\
\text { de anuncios para } \\
\text { escribir el anuncio }\end{array}$ & $\begin{array}{l}\text { El examinando } \\
\text { deberá redactar } \\
\text { un anuncio breve } \\
\text { para colocar en } \\
\text { un tablón de } \\
\text { anuncios } \\
\text { + acorde con la } \\
\text { finalidad / con- } \\
\text { texto indicado e } \\
+ \text { incluyendo } \\
\text { la información } \\
\text { solicitada }\end{array}$ & $\begin{array}{l}\text { El examinando PUEDE: } \\
\text { Redactar un anuncio muy } \\
\text { sencillo, relacionado con el } \\
\text { ámbito laboral, público o } \\
\text { personal, } \\
\text { + ofreciendo la información } \\
\text { solicitada } \\
\text { + cumpliendo los fines } \\
\text { funcionales } \\
\text { + manifestando adecuación } \\
\text { al género textual. } \\
\text { + utilizando frases y ora- } \\
\text { ciones breves y sencillas, } \\
\text { enlazadas con conectores } \\
\text { muy básicos }\end{array}$ \\
\hline
\end{tabular}

Figura 2. Tarea 2 de la Prueba de expresión e interacción escritasł anuncio Tarea 2

Usted busca trabajo.

V Escriba un anuncio. El anuncio debe explicar:

- Tipo de trabajo que busca

- Su experiencia

- Horario y disponibilidad

- Forma de contrato

TABLÓN DE ANUNCIOS

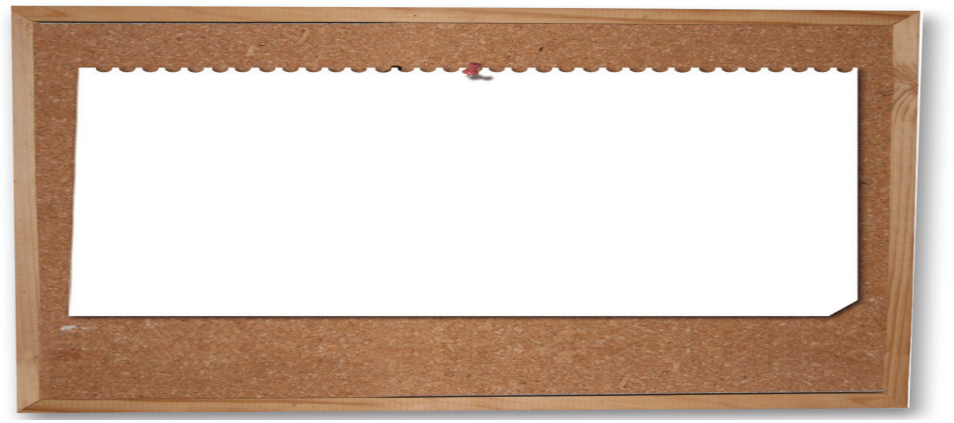


La Tarea 3 consiste en redactar un correo electrónico en respuesta a un mensaje recibido, pero no se muestran aquí por limitaciones de espacio. Se pueden consultar un examen completo en www.diplomaletra.com

Las escalas de evaluación holística para las pruebas de expresión e interacción escritas se han establecido teniendo en cuenta 4 (cuatro) niveles de calificación. Posteriormente se ha transformado en su equivalencia a una puntuación de 0 a 10 debido a que la población está más familiarizada con esta escala numérica y puede valorar mejor la calificación obtenida. Se han tenido en cuenta dos aspectos de manera global, la eficacia comunicativa y el control lingüístico integrando léxico y gramática. En estas pruebas también se ha tenido en cuenta la legibilidad de la letra como elemento esencial para la evaluación:

\section{Respuesta muy satisfactoria (calificación: 4)}

+ Comunicación - Redacta muy satisfactoriamente el texto requerido, ofreciendo más información pertinente de la solicitada para cumplir los fines funcionales. Se adecua al género textual. Se comprende sin necesidad de releer.

+ Control lingüístico - Utiliza un repertorio lingüístico variado y correcto, dentro de las limitaciones propias del nivel.

\section{Respuesta satisfactoria (calificaciónः 3)}

+ Comunicación - Redacta de manera satisfactoria el texto requerido, ofreciendo información suficiente para cumplir los fines funcionales propuestos. Se adecua al género textual. Se puede comprender aunque sea necesaria una relectura.

+ Control lingüístico - Utiliza un repertorio de recursos lingüísticos básico pero suficiente, aunque con cierta dificultad.

Respuesta insatisfactoria (calificación: 2)

- Comunicación - Redacta el texto requerido con seria dificultad, sin satisfacer la necesidad comunicativa planteada, ya que no ofrece la información suficiente para cumplir con los fines funcionales. No se adecua al género textual. No se puede comprender ni siquiera con una relectura.

+ Control lingüístico - Utiliza con mucha dificultad un repertorio insuficiente de recursos léxicos y gramaticales.

\section{Respuesta muy insatisfactoria (calificación: 1 )}

+ Comunicación - El candidato es incapaz de redactar el texto requerido.

+ Control lingüístico - Prácticamente nulo. 
A continuación se muestran dos ejemplos reales de candidatos que han obtenido la puntuación mínima y la máxima en la evaluación holística de la Tarea 2:

\section{TABLÓN DE ANUNCIOS}

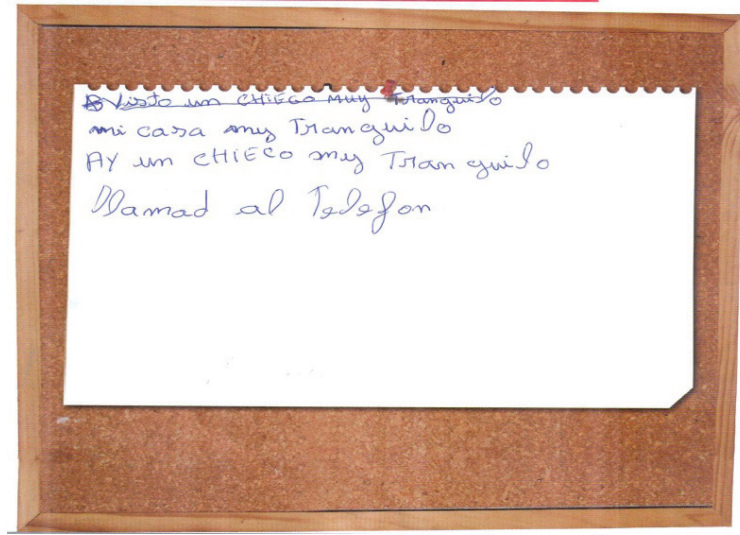

\section{TABLÓN DE ANUNCIOS}

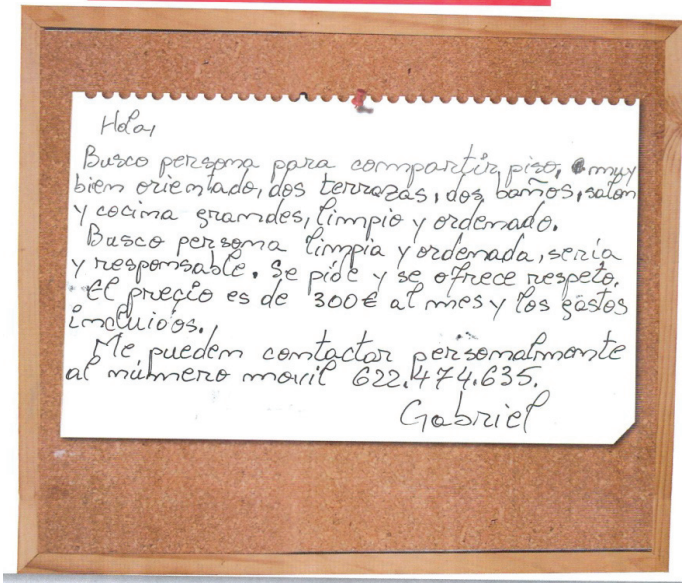

Las escalas de evaluación analítica para las pruebas de expresión e interacción escritas también se han configurado en una escala de 4 puntos, convertibles en calificación final del Examen en una escala de 0 a 10 por las razones anteriormente mencionadas. Los 6 aspectos de la expresión y la interacción escritos son los que se reproducen aquí de la hoja del evaluador analítico: 


\begin{tabular}{|c|c|c|c|c|}
\hline I. EXPRESIÓN E INTERACCIÓN GLOBAL & 1 & 2 & 3 & 4 \\
\hline $\begin{array}{l}\text { Transmite y solicita información por escrito de forma sencilla en situacio- } \\
\text { nes concretas de los ámbitos personal, administrativo y laboral. Cumple } \\
\text { los fines funcionales (intención comunicativa) escribiendo la información } \\
\text { pertinente y requerida. Se entiende el mensaje. }\end{array}$ & & & & \\
\hline 2. ORGANIZACIÓN Y COHESIÓN / COHERENCIA DISCURSIVA & 1 & 2 & 3 & 4 \\
\hline $\begin{array}{l}\text { Organiza el texto de manera elemental y lógica adecuada al género textual } \\
\text { requerido. Usa conectores básicos para enlazar palabras y signos de pun- } \\
\text { tuación (puntos, comas) para separar las ideas. }\end{array}$ & & & & \\
\hline 3. Competencia sociopragmática & & & & \\
\hline $\begin{array}{l}\text { Utiliza un registro adecuado a la situación de comunicación, a la intención } \\
\text { comunicativa y al género textual. }\end{array}$ & & & & \\
\hline 4. Alcance y control de vocabulario & 1 & 2 & 3 & 4 \\
\hline $\begin{array}{l}\text { Dispone de un repertorio básico de palabras y frases sencillas relativas a } \\
\text { sus datos personales y a situaciones concretas de los ámbitos administra- } \\
\text { tivo y laboral. }\end{array}$ & & & & \\
\hline 5. AlCANCE y CONT Rol gramatical & 1 & 2 & 3 & 4 \\
\hline $\begin{array}{l}\text { Muestra un control limitado de unas pocas estructuras gramaticales sen- } \\
\text { cillas y de modelos de oraciones dentro de un repertorio básico o apren- } \\
\text { dido, que le permiten expresar su intención comunicativa, aunque con } \\
\text { errores sistemáticos propios del nivel. }\end{array}$ & & & & \\
\hline 6. Control ortográfico & 1 & 2 & 3 & 4 \\
\hline $\begin{array}{l}\text { Escribe con letra legible en mayúsculas y minúsculas. No se tendrán en } \\
\text { cuenta los errores ortográficos. }\end{array}$ & & & & \\
\hline
\end{tabular}

Es interesante destacar que en la evaluación ha primado el constructo de eficacia comunicativa más que el de corrección lingüística y que, siguiendo el MCRE en toda la actuación de los candidatos se han tenido en cuenta las competencias no lingüísticas del saber ser y hacer, en el sentido de uso de habilidades, actitudes y estrategias que faciliten la comunicación, tanto en la interacción escrita como en la oral.

\section{Prueba de expresión e interacción orales}

Aplicando los mismos criterios que los expuestos para la Prueba escrita, en esta Prueba de desempeño de interacción oral se han intentado controlar todas las variables que pudieran incidir en los resultados de la conducta comunicativa del candidato. La fiabilidad del diseño de la Prueba y la validez del constructo comunicativo se ha pilotado con casi 200 inmigrantes hablantes no nativos de español, seleccionados por su nivel inicial de lengua en los 20 centros colaboradores con los que se ha trabajado durante todo el periodo de investigación de este proyecto. La instrucción realizada con los entrevistadores, quienes actuaban como evaluadores holísticos y con los evaluadores analíticos, que actuaban como observado- 
res discretos durante la prueba se dirigía a que actuaran en todo momento como un interlocutor cooperativo, que hablara despacio y con claridad, sin modismos ni frases hechas.

Esta Prueba tiene una duración de tiempo de 8-10 minutos. En todos los casos se ha solicitado el permiso del candidato para grabar la prueba y se conservan las grabaciones de la mayoría de los candidatos de las pruebas de pilotaje y de los 300 candidatos de la primera convocatoria del Examen LETRA. Los resultados se están estudiando en investigaciones focalizadas en diferentes aspectos de la competencia comunicativa. Las especificaciones de la Prueba se encuentran publicadas en Baralo y Estaire (2011).

La prueba de expresión e interacción orales simula una entrevista de trabajo con tres partes diferenciadas pero sin ninguna interrupción. La conversación es sostenida en todo momento por el entrevistador, que toma la iniciativa, colabora, asiente, es decir, se comporta como un hablante real en una conversación nativa, pero manteniendo el lenguaje facilitador, de ritmo más lento que el habitual, con una sintaxis simple, sin usar frases idiomáticas, tratando de hacerlo sentirse cómodo y relajado al candidato, ya tenso por la propia situación de examen.

En la primera parte el candidato debe participar en una entrevista para hablar de los datos personales y las experiencias e intereses laborales del candidato.

En la segunda parte debe mantener un monólogo sostenido, en el que el examinando describe una lámina representativa del ámbito laboral. Se espera que pueda participar en una conversación cara a cara con el examinador acerca de ciertos aspectos relacionados con la misma. Se han diseñado 40 láminas correspondientes a otras tantas profesiones en las que suelen participar los trabajadores inmigrantes, según la ENI (2007). Las láminas contienen información referencial sobre el ámbito del trabajo, con una foto de un trabajador, con un ambiente siempre dinámico y positivo. En la parte derecha se incluyen instrumentos propios de su trabajo, así como referencias a tiempos, horarios, dificultades, riesgos laborales, de manera que se pueda facilitar la evaluación del alcance léxico específico de la profesión elegida, con medición del vocabulario referido a las nociones generales y específicas representadas.

El candidato tiene la oportunidad, unos minutos antes de comenzar la entrevista, y guiado por una persona especializada de la organización del Examen, de seleccionar la lámina con la que se sienta más cómodo porque conoce y se identifica con la profesión elegida, sea porque está trabajando en ella o porque tiene la experiencia de su país en ese tipo de trabajo. 
A continuación se ofrece una muestra del estímulo visual que sirve de soporte a esta parte del monólogo sostenido de la expresión y la interacción orales, de peluquería y de la construcción:
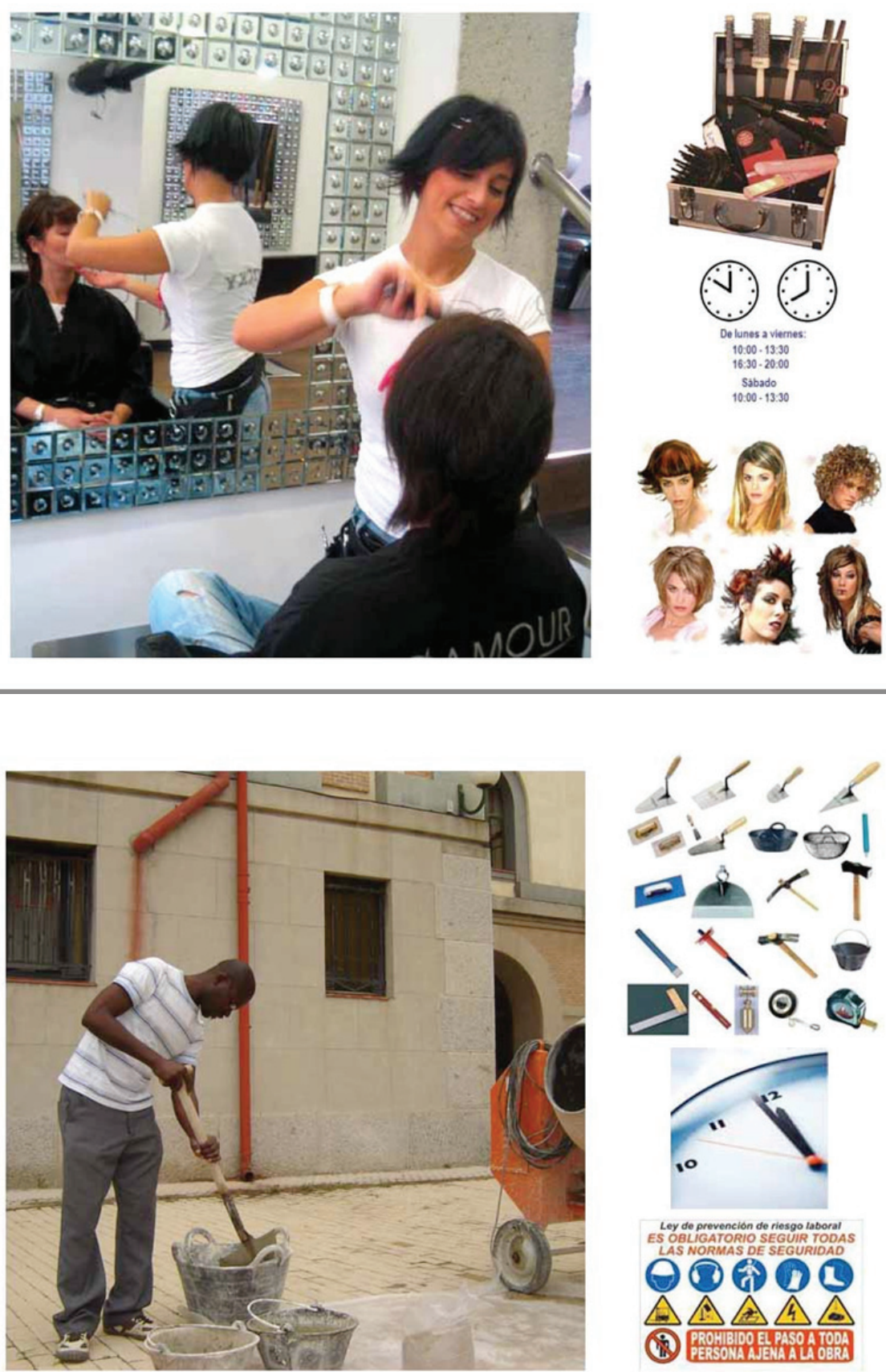

Ley de prevencion de riesgo laboral ES OBLIGATORIO SEGUIR TODAS
LAS NORMAS DE SEGURIDAD Q) 120 $\Delta \triangle \Delta \triangle \triangle$ (2) RROHIIIDO ELPASOA TODA 
En la tercera y última parte de la Prueba de expresión e interacción orales, que es la última parte de la entrevista, el candidato participa en una conversación cara a cara con el examinador acerca del contenido de una lámina relacionada con el ámbito administrativo-laboral y con algunos documentos administrativos. Se han utilizado como estímulo visual fotos de pasaportes, tarjetas sanitarias, tarjeta de residente, documento acreditativo de la prestación por desempleo, para que el entrevistador pueda preguntarle acerca de esa documentación, de las instituciones o lugares en los que solicita, o se utiliza, de los trámites administrativos o laborales que se realizan con ellos.

Las Escalas de evaluación holística para las pruebas de expresión e interacción orales se han diseñado, como en la prueba escrita, con tres criterios: comunicación, relación con el interlocutor y control lingüístico, sobre los cuales el entrevistador, que funciona como evaluador holístico, califica en una escala de 1 a 4 . Las especificaciones han sido validadas y utilizadas como herramienta imprescindible para la formación de los evaluadores y como manera de conseguir el acuerdo entre jueces. Cada una de las tres partes de la prueba se califica utilizando la escala que se presenta a continuación. La puntuación para cada parte se obtiene siguiendo las tablas de conversión acordadas.

\begin{tabular}{|c|}
\hline RESPUESTA MUY SATISFACTORIA (CALIFICACIÓNः 4) \\
\hline $\begin{array}{l}\text { Comunicación - El candidato cumple muy adecuadamente y con cierta soltura los ob- } \\
\text { jetivos comunicativos de la prueba. La sencillez de su mensaje es claramente suficiente } \\
\text { para satisfacer la necesidad comunicativa planteada. }\end{array}$ \\
\hline $\begin{array}{l}\text { Relación con el interlocutor - Manifiesta un grado incipiente de independencia del in- } \\
\text { terlocutor, con capacidad de iniciar algún intercambio o de ofrecer información no so- } \\
\text { licitada. Un interlocutor acostumbrado a tratar con hablantes de otras lenguas puede } \\
\text { comprenderle sin mayor esfuerzo. }\end{array}$ \\
\hline $\begin{array}{l}\text { Control lingüístico - Dentro de las limitaciones de un nivel A2-1 y de un repertorio } \\
\text { reducido, el candidato utiliza los recursos lingüísticos básicos a su alcance con cierta } \\
\text { soltura, aunque con algunos errores propios del nivel. }\end{array}$ \\
\hline Respuesta satisfactoria (CALIficACión: 3) \\
\hline $\begin{array}{l}\text { Comunicación - El candidato cumple adecuadamente los objetivos comunicativos de } \\
\text { la prueba aunque evidenciando ocasionalmente un cierto grado de dificultad. A pesar } \\
\text { de esta dificultad, su mensaje es suficiente para satisfacer la necesidad comunicativa } \\
\text { planteada. }\end{array}$ \\
\hline $\begin{array}{l}\text { Relación con el interlocutor - Para su actuación, el candidato depende ocasionalmente de } \\
\text { la cooperación del interlocutor. Un interlocutor acostumbrado a tratar con hablantes de } \\
\text { otras lenguas puede comprenderle con cierto esfuerzo y concentración. }\end{array}$ \\
\hline
\end{tabular}


Control lingüístico - Manifiesta cierta dificultad en la utilización de los recursos lingüísticos básicos de un nivel A2-n y correspondientes a un repertorio reducido: necesita algunas pausas y reformulaciones, a menudo demuestra dudas y comete numerosos errores, la mayoría propios de su nivel.

Respuesta insatisfactoria (CAlificación: 2)

Comunicación - El candidato tiene serias dificultades para reaccionar comunicativamente a la situación planteada. En algunas ocasiones su mensaje se relaciona sólo vagamente con dicha situación, no satisfaciendo por tanto la necesidad comunicativa planteada.

Relación con el interlocutor - Para su actuación, el candidato depende totalmente de la cooperación del interlocutor. Un interlocutor acostumbrado a tratar con hablantes de otras lenguas necesita esforzarse al máximo para comprenderle.

Control lingüístico - Utiliza de forma muy limitada y poco controlada los recursos lingüísticos básicos de un nivel A2-n y correspondientes a un repertorio reducido: de forma continua comete errores, necesita pausas y reformulaciones y demuestra dudas.

Respuesta muy insatisfactoria (CALIFICACión: I)

Relación con el interlocutor - La cooperación del interlocutor no es suficiente para establecer la comunicación con el candidato. Aún para un interlocutor acostumbrado a tratar con hablantes de otras lenguas es prácticamente imposible comprenderle.

Control lingüístico - Prácticamente nulo.

Las tablas con las especificaciones para la evaluación analítica de las 3 tareas de expresión e interacción orales se encuentran publicadas en Baralo y Estaire (2011). Aquí solo indicamos los 10 aspectos que el observador que actúa como evaluador analítico debe tener en cuenta en cada una de las partes de su interacción comunicativa:

\section{INTERACCión GLOBAL I}

Participa en una conversación de forma sencilla para describir acciones y elementos de los ámbitos personal, laboral y administrativo. Se ayuda de estrategias compensatorias para la comprensión y la producción.

\section{INTERACCIÓN GLOBAL II}

Se hace entender y cumple adecuadamente los fines funcionales (la intención comunicativa) con un cierto grado de independencia del interlocutor.

\section{Fluidez}

Se desenvuelve con enunciados breves, con pausas para buscar expresiones, articular palabras menos habituales, reformular y salvar la comunicación. 


\section{Organización y COHESIón/COHERENCia discursiva}

Organiza su discurso de manera adecuada y lógica para describir acciones y elementos de los ámbitos personal, laboral y administrativo.

\section{Competencia sociopragmática}

Establece contactos sociales básicos en un registro formal manifestando cortesía (empleo de expresiones de disculpa, agradecimiento, petición) y utilizando formas de tratamiento y saludos apropiados.

\section{Alcance y control de vocabulario}

Domina un repertorio básico de palabras y frases sencillas relativas a sus datos personales y a situaciones concretas de los ámbitos administrativo y laboral (horarios y herramientas de una profesión, riesgos laborales que entraña y buenas prácticas en el trabajo) que le permite aportar la información requerida.

\section{Alcance y control gramatical}

Muestra un control limitado de unas pocas estructuras gramaticales sencillas y de modelos de oraciones dentro de un repertorio básico o aprendido, que le permiten expresar su intención comunicativa (afirmativa, negativa, interrogativa, exhortativa), aunque con errores sistemáticos propios del nivel.

\section{Control fonológico I}

Su pronunciación está marcada por el acento extranjero, pero es comprensible. Deletrea de forma suficientemente clara para ser comprendido.

\section{Control fonológico II}

La entonación es adecuada a su intención comunicativa y discrimina la intención comunicativa del interlocutor por la entonación que emplea.

\section{io. Referentes socioculturales}

Es capaz de identificar instituciones/oficinas del ámbito administrativo-laboral y centros de apoyo a las actividades del trabajador inmigrante como agente social. Es capaz de explicar su función así como algunas gestiones que se realizan en los mismos. Es capaz de identificar documentos pertinentes para algunas de esas u otras gestiones, explicar su función y los procedimientos para su obtención.

El lector atento habrá podido comprobar que los criterios de evaluación propuestos tienen una clara tendencia comunicativa, ya que los cinco primeros 
criterios que se valoran tienen que ver con la eficacia y adecuación comunicativa. El mismo objetivo de evaluación comunicativo lo tienen el criterio 9, que se refiere a la entonación relacionada con la intención del hablante y el criterio 10 , vinculado al conocimiento de los ámbitos laborales y administrativos. Solo dos criterios de evaluación de los diez enunciados tienen que ver con el dominio del sistema lingüístico de forma específica, pero tanto el alcance gramatical como la pronunciación están vinculados también al logro de la eficacia comunicativa, pues la gramática se supedita a la intención comunicativa y la pronunciación a que se puedan distinguir los elementos léxicos. El alcance léxico de cada candidato se ha personalizado en cuanto a su propia actividad laboral mediante el procedimiento de elegir la lámina que servirá de soporte y de estímulo visual para su monólogo sostenido y la interacción con el examinador/entrevistador.

\section{Conclusiones}

Después de esta somera presentación del diseño de las pruebas de expresión y de interacción escritas y orales pueden quedar más al descubierto o pueden hacerse más conscientes las dificultades que ofrece la elaboración de unas tareas que sean eficaces para conseguir asegurar una evaluación subjetiva justa, igualitaria, que aseguren un alto grado de validez, de fiabilidad y de viabilidad. La validez se ha conseguido con la comprobación de que estas pruebas realmente permiten al candidato realizar actividades comunicativas que permiten evaluar el constructo del examen, es decir, su nivel de dominio lingüístico comunicativo, al menos de un A2-n en lengua española. La fiabilidad se ha podido conseguir mediante la formación sistemática de los evaluadores participantes, ya que se han realizado cursos para los 40 participantes en la convocatoria del examen, con unas 100 horas de formación, incluyendo las sesiones de acuerdo entre jueces. La viabilidad ha quedado demostrada por las propias convocatorias realizadas, con una tasa de aprobados superior al $80 \%$ y el interés despertado por la obtención de este Diploma LETRA tanto por los trabajadores inmigrantes como por los Centros que se encargan, entre otras tareas, de enseñarles la lengua. Hemos expuesto las decisiones tomadas para la evaluación de la expresión e interacción escritas y orales siendo conscientes de que la calificación depende en parte del propio evaluador, que actúa de manera subjetiva frente al candidato y a las variadas condiciones y factores individuales y contextuales que pueden influir. De ahí la importancia crucial del uso de instrumentos como las especificaciones de las pruebas y los descriptores para cada uno de los niveles de calificación.

El diseño aquí presentado se ha sometido a un comité de expertos externos, teniendo en cuenta los estándares de calidad de ALTE y de EALTE; se han pro- 
cesado estadísticamente los resultados para comprobar la validez y la fiabilidad de las pruebas y se ha realizado la implantación del examen con una $1^{\text {a }}$ convocatoria en noviembre de 2010 para 300 candidatos y una $2^{\text {a }}$ convocatoria en noviembre de 2011. Los resultados han sido muy satisfactorios por el interés de los candidatos y los altos porcentajes de aprobados.

\section{Bibliografía}

[AlTE] The Association of Language Testers in Europe. [Disponible en: <http://www.alte.org/>].

Baralo, M. y Estaire, S. (2011). «Variables socioculturales y comunicativas en el diseño curricular de una certificación de español para trabajadores inmigrantes». Lengua y migración. 3, vol. 2:5-41.

Bordón, T. (2006). La evaluación de la lengua en el marco del E/L2: bases y procedimientos. Madrid. Arcolibros.

Consejería de Inmigración y Cooperación (2009). Plan de integración 2009-2010 de la Comunidad de Madrid. [Disponible en:<http://www.madrid.org/inmnigramadrid $>$ ].

Consejo de Europa (2002). Marco común europeo de referencia para las lenguas: enseñanza, aprendizaje y evaluación. Madrid. MEC y Anaya. Versión española en Centro virtual Cervantes, Instituto Cervantes [Disponible en: <http // cvc.cervantes,es/obref/marco/default.htm $>$ ].

Escudero, I. y Guerra, M. ${ }^{a}$ R. (2009). «La observación etnográfica como método para el análisis de necesidades comunicativas de la población inmigrante». Actas del XVII Congreso Internacional de AESLA. Universidad de Castilla La Mancha.

Estaire, S. (2009). El aprendizaje mediante tareas: de la programación al aula. Madrid. Edinumen.

[EALTA] European Association for Language Testing and AssessMENT. En: <www.ealta.eu.org/>.

Instituto Cervantes (2006). Plan Curricular del Instituto Cervantes. Madrid. Instituto Cervantes. Biblioteca Nueva.

Instituto Nacional de Estadística (2007). Encuesta Nacional de Inmigrantes 2007. [Disponible en: <http://www.ine.es/prodyser/pubweb/eni07/ eni07.pdf>]. 
Martín Leralta, S. (2009). «Certificación lingüística de nivel inicial para inmigrantes en contexto laboral: ejemplo de una prueba del examen DILE». Lengua y migración. 3. vol. 1: 89-104.

- (2011). «La evaluación de la interacción oral para la certificación lingüística en L2». En: Actas XVI Congreso Internacional de la ALFAL. Universidad de Alcalá. Alcalá de Henares.

Martínez Baztám, A. (2008). La evaluación oral: equivalencia entre las guidelines de ACTFL y algunas escalas del MCER. Tesis doctoral. Dir. Calderón Campos, Miguel Universidad de Granada.

- (2011). La evaluación de las lenguas: garantías y militaciones. Andalucía. Octaedro.

Moreno Fernández, F. (2009). «Integración sociolingüística en contextos de inmigración: marco epistemológico para su estudio en España». Lengua y migración. vol. 1-1: 121-156.

Otero Roth, J. (2007). «Lengua y migraciones: aspectos culturales de la inmigración latinoamericana en España». Análisis del Real Instituto Elcano (ARI). No 36/. Madrid. Real Instituto El Cano. Área: Lengua y Cultura. [Disponible en: <http //www.realinstitutoelcano.org/wps/portal/rielcano/ contenido?WCM_GLOBAL_CONTEXT=/wps/wcm/connect/elcano/ Elcano_es/Zonas_es/ARI+36-2007>].

Sidro Gual, A. (2011). La expresión e interacción escrita en la certificación lingüística para inmigrantes: análisis de producciones y actividades didácticas. Trabajo final del Máster en Lingüística Aplicada a la Enseñanza de Español como Lengua Extranjera. Madrid. Universidad Nebrija.

YAGÜE, A. (2010). «Examinando exámenes. Consideraciones acerca de las pruebas de medición en ELE». marcoELE, revista de didáctica de ELE. 10 [Disponible en: <http://marcoele.com/descargas/10/yague_examinandoexamenes.pdf $>$ ]. 